\title{
Rough Computational Approach to UAR based on Dominance Matrix in IOIS
}

\author{
Xiaoyan Zhang, Weihua $\mathrm{Xu}^{1}$ \\ Chongqing University of Technology, Chongqing, China \\ E-mail: zhangxyms@gmail.com, chxuwh@gmail.com \\ Received March 28, 2011; revised June 30, 2011; accepted July 7, 2011
}

\begin{abstract}
Rough set theory is a new mathematical tool to deal with vagueness and uncertainty. The classical rough set theory based on equivalence relation has made a great progress, while the equivalence relation is too harsh to meet and is extended to dominance relation in real world. It is important to investigate rough computational methods for rough set theory, which is one of the bottleneck problems in the development of rough set theory. In this article, rough computational approach to upper approximation reduction (UAR) is discussed based on dominance matrix in inconsistent ordered information systems (IOIS). The algorithm of upper approximation reduction is obtained, from which we can provide approach to upper approximation reduction operated simply in inconsistent systems based on dominance relations. Finally, an example illustrates the validity of this method, and shows the method is excellent to a complicated information system.
\end{abstract}

Keywords: Dominance Relation, Information System, Rough Set, Upper Approximation Reduction

\section{Introduction}

The rough set theory, proposed by Pawlak in the early 1980s [1], is an extension of the classical set theory for modeling uncertainty or imprecision information. The research has recently roused great interest in the theoretical and application fronts, such as machine learning, pattern recognition, data analysis, and so on.

Attributes reduction is one of the hot research topics of rough set theory. Much study on this area had been reported and many useful results were obtained until now [2-7]. However, most work was based on consistent information systems, and the main methodology has been developed under equivalence relations (indiscernibility relations). In practice, most of information systems are not only inconsistent, but also based on dominance relations because of various factors. The ordering of properties of attributes plays a crucial role in those systems. For this reason, Greco, Matarazzo, and Slowinski [8-13] proposed an extension rough sets theory, called the dominance-based rough sets approach (DRSA) to take into account the ordering properties of attributes. This innovation is mainly based on substitution of the indiscernibility relation by a dominance relation. In DRSA, where condition attributes and classes are preference ordered. And many studies have been made in DRSA [14-19]. But simpler results of knowl- edge reductions are very poor in inconsistent ordered information systems until now.

In this paper, the method operated simply for upper approximation reduction is introduced in inconsistent is obtained, from which we can provide new approach to knowledge reductions in inconsistent systems based on dominance relations. Finally, an example illustrates the validity of this method, and shows the method is excellent to a complicated information system.

\section{Rough Sets and OIS}

The following recalls necessary concepts and preliminaries required in the sequel of our work. Detailed description of the theory can be found in $[4,5]$.

An information system with decisions is an ordered quadruple $\mathcal{I}=(U, A \cup D, F, G)$, where

$U=\left\{x_{1}, x_{2}, \cdots, x_{n}\right\}$ is a non-empty finite set of objects;

$A \cup D$ is a non-empty finite attributes set;

$A=\left\{a_{1}, a_{2}, \cdots, a_{p}\right\}$ denotes the set of condition attributes;

$D=\left\{d_{1}, d_{2}, \cdots, d_{q}\right\}$ denotes the set of decision attributes, and $A \cap D=\phi$;

$F=\left\{f_{k} \mid U \rightarrow V_{k}, k \leq p\right\}, f_{k}(x)$ is the value of $a_{k}$ on $x \in U, V_{k}$ is the domain of $a_{k}, a_{k} \in A$; 
$G=\left\{g_{k^{\prime}} \mid U \rightarrow V_{k^{\prime}}, k^{\prime} \leq q\right\}, g_{k^{\prime}}(x)$ is the value of $d_{k^{\prime}}$ on $x \in U, V_{k^{\prime}}$ is the domain of $d_{k^{\prime}}, d_{k^{\prime}} \in D$.

In an information system, if the domain of a attribute is ordered according to a decreasing or increasing preference, then the attribute is a criterion.

Definition 2.1 (See [4]) An information system is called an ordered information system (OIS) if all condition attributes are criterions.

Assumed that the domain of a criterion $a \in A$ is complete pre-ordered by an outranking relation $\succeq_{a}$, then $x \succeq_{a} y$ means that $x$ is at least as good as $y$ with respect to criterion $a$. And we can say that $x$ dominates $y$. In the following, without any loss of generality, we consider condition and decision criterions having a numerical domain, that is, $V_{a} \subseteq \mathcal{R}$ ( $\mathcal{R}$ denotes the set of real numbers).

We define $x \succeq y$ by $f(x, a) \geq f(y, a)$ according to increasing preference, where $a \in A$ and $x, y \in U$. For a subset of attributes $B \subseteq A, x \succeq_{B} y$ means that $x \succeq_{a} y$ for any $a \in B$. That is to say $x$ dominates $y$ with respect to all attributes in $B$. Furthermore, we denote $x \succeq_{B} y$ by $x R_{B}^{\geq} y$. In general, we indicate a ordered information systems with decision by

$\mathcal{I}^{\succeq}=(U, A \cup D, F, G)$.

Thus the following definition can be obtained.

Let $\mathcal{I}^{\succeq}=(U, A \cup D, F, G)$ be an ordered information system with decisions, for $B \subseteq A$, denote

$$
\begin{gathered}
R_{B}^{\succ}=\left\{\left(x_{i}, x_{j}\right) \in U \times U \mid f_{l}\left(x_{i}\right) \geq f_{l}\left(x_{j}\right), \forall a_{l} \in B\right\} \\
R_{\bar{D}}^{\succ}=\left\{\left(x_{i}, x_{j}\right) \in U \times U \mid g_{m}\left(x_{i}\right) \geq g_{m}\left(x_{j}\right), \forall d_{m} \in D\right\}
\end{gathered}
$$

$R_{B}^{\succ}$. and $R_{D}^{\succ}$ are called dominance relations of information system $\mathcal{I}^{\succeq}$.

If we denote

$$
\begin{gathered}
{\left[x_{i}\right]_{B}^{\succ}=\left\{x_{j} \in U \mid\left(x_{j}, x_{i}\right) \in R_{B}^{\succ}\right\}} \\
\left\{x_{j} \in U \mid f_{l}\left(x_{j}\right) \geq f_{l}\left(x_{i}\right), \forall a_{l} \in B\right\} ; \\
{\left[x_{i}\right]_{D}^{\succ}=\left\{x_{j} \in U \mid\left(x_{j}, x_{i}\right) \in R_{D}^{\succ}\right\}} \\
\left\{x_{j} \in U \mid g_{m}\left(x_{j}\right) \geq g_{m}\left(x_{i}\right), \forall d_{m} \in D\right\},
\end{gathered}
$$

then the following properties of a dominance relation are trivial.

Proposition 2.1 (See [4]) Let $R_{A}^{\succ}$ be a dominance relation. The following hold.

1) $R_{A}^{\succ}$ is reflexive, transitive, but not symmetric, so it is not a equivalence relation.

2) If $B \subseteq A$, then $R_{A}^{\succ} \subseteq R_{B}^{\succ}$.

3) If $B \subseteq A$, then $\left[x_{i}\right]_{A}^{\succeq} \subseteq\left[x_{i}\right]_{B}^{\succ}$.

4) If $x_{j} \in\left[x_{i}\right]_{A}^{\succ}$, then $\left[x_{j}\right]_{A}^{\succeq} \subseteq\left[x_{i}\right]_{A}^{\succ}$ and

$$
\left[x_{i}\right]_{A}^{\succ}=\cup\left\{\left[x_{j}\right]_{A}^{\succ} \mid x_{j} \in\left[x_{i}\right]_{A}^{\succeq}\right\} .
$$

$$
\text { 5) }\left[x_{j}\right]_{A}^{\succeq}=\left[x_{i}\right]_{A}^{\succeq} \text { iff }
$$

$f\left(x_{i}, a\right)=f\left(x_{j}, a\right)(\forall a \in A)$.

6) $\mathcal{J}=\cup\left\{[x]_{A}^{\succeq} \mid x \in U\right\}$ constitute a covering of $U$.

For any subset $X$ of $U$, and $A$ of $\mathcal{I}^{\succeq}$ define

$$
\begin{gathered}
R_{A}^{\succ}(X)=\left\{x \in U \mid[x]_{A}^{\succ} \subseteq X\right\} ; \\
\overline{R_{A}^{\succ}}(X)=\left\{x \in U \mid[x]_{A}^{\succ} \cap X \neq \phi\right\}
\end{gathered}
$$

$R_{A}^{\succ}(X)$ and $\overline{R_{A}^{\succ}}(x)$ are said to be the lower and upper approximation of $X$ with respect to a dominance relation $R_{A}^{\succ}$. And the approximations have also some properties which are similar to those of Pawlak approximation spaces.

Definition 2.2 (See [4]) For a ordered information system with decisions $\mathcal{I}^{\succeq}=(U, A \cup D, F, G)$, if $R_{A} \subseteq R_{D}^{\succ}$, then this information system is consistent, otherwise, this information system is inconsistent.

For simple description, the following information systems with decisions are based on dominance relations, i.e. ordered information systems.

Let $\mathcal{I}^{\succeq}=(U, A \cup D, F, G)$ be an inconsistent ordered information system, and denote

$$
\begin{gathered}
D_{k} \in U / R_{D}^{\succ}, \quad(k \in\{1,2, \cdots, r\}) \\
\overline{\eta_{B}}=\left(\overline{R_{B}^{\succ}}\left(D_{1}\right), \overline{R_{B}^{\succcurlyeq}}\left(D_{2}\right), \cdots, \overline{R_{B}^{\succ}}\left(D_{r}\right)\right)
\end{gathered}
$$

the following definition is gave.

Definition 2.3 (See [5]) If $\eta_{B}=\overline{\eta_{A}}$, for all $B \subseteq A$, we say that $B$ is an upper approximation consistent set of $\mathcal{I}^{\succeq}$. If $B$ is an upper approximation consistent set, and no proper subset of $B$ is upper approximation consistent set, then $B$ is called an upper approximation consistent reduction of $\mathcal{I}^{\succeq}$.

From the above, we can find that an upper approximation consistent set preserves the upper approximation of every decision class.

Theorem 2.1 (See [5]) Let $\mathcal{I}^{\succeq}=(U, A \cup D, F, G)$ be an information system, $B \subseteq A$, then $B$ is an upper approximation consistent set if and only if there exist $b \in B$ such that $f_{b}(x)>f_{b}(y)$ when $x \notin \overline{R_{A}^{\succ}}\left(D_{k}\right)$ and $y \in R_{A}^{\succ}\left(D_{k}\right)$ for every

$$
D_{k} \in U / R_{D}^{\succ} \quad(k=1,2, \cdots, r) .
$$

From the theorem, authors have provided approach to upper approximation reduction in inconsistent ordered systems based on indiscernable matrixes in [5]. We can find that it is not convenient to use the method by computers. So we will propose the approach to upper approximation reduction operated simply by computers. 


\section{Rough Computational Approach to UAR Based on Dominance Matrix}

\subsection{Dominance Matrices and Upper Approximation Decision Matrices}

In this section, the dominance matrices and upper approximation decision matrices are proposed, and some properties are obtained.

Definition 3.1 Let $\mathcal{I}^{\succeq}=(U, A \cup D, F, G)$ be an ordered information system, and denote

$$
M_{B}=\left(m_{i j}\right)_{n \times n},
$$

where

$$
m_{i j}= \begin{cases}1, & x_{j} \in\left[x_{i}\right]_{B}^{\succ}, \\ 0, & \text { otherwise. }\end{cases}
$$

The matrix $M_{B}$ is called dominance matrix of attributes set $B \subseteq A$. If $|B|=l$, we say that the order of $M_{B}$ is $l$.

Definition 3.2 Let $\mathcal{I}^{\succeq}=(U, A \cup D, F, G)$ be an ordered information system, and dominance matrices $M_{B}$, $M_{C}$ of attributes sets $B, C \subseteq A$. The intersection of $M_{B}$ and $M_{C}$ is defined by

$$
M_{B} \cap M_{C}=\left(m_{i j}\right)_{n \times n} \cap\left(m_{i j}\right)_{n \times n}=\left(\min \left\{m_{i j}, m_{i j}\right\}\right)_{n \times n} .
$$

The following properties are obviously.

Proposition 3.1 Let $M_{B}, M_{C}$ be dominance matrices of attributes sets $B, C \subseteq A$, the following results always hold.

1) $m_{i i}=1$.

2) If $M_{B}, M_{C}$, then $M_{B \cup C}=M_{B} \cap M_{C}$.

Definition 3.3 Let $\mathcal{I}^{\succeq}=(U, A \cup D, F, G)$ be an ordered information system, and denote

where

$$
M_{D}=\left(r_{i j}\right)_{n \times n},
$$

$$
r_{i j}= \begin{cases}0, & x_{i} \notin \overline{R_{A}^{\succ}}\left(D_{k_{0}}\right) \text { and } x_{j} \in \overline{R_{A}^{\succ}}\left(D_{k_{0}}\right) \text { hold } \\ & \text { at same time for some } k_{0} \in\{1,2, \cdots, r\} . \\ 1, & \text { otherwise. }\end{cases}
$$

The matrix $M_{D}$ is called upper approximation decision matrix of $\mathcal{I}^{\succeq}$.

From the above, we can see that the dominance relation of objects is decided by dominance matrices, and different decisions of objects is decided by upper approximation decision matrix.

Definition 3.4 Let $\alpha=\left(a_{1}, a_{2}, \cdots, a_{n}\right)$ and $\beta=$ $\left(b_{1}, b_{2}, \cdots, b_{n}\right)$ be two $n$ dimension vectors. If $a_{i} \leq b_{i},(i=1,2, \cdots, n)$, we say vector $\alpha$ is less than vector $\beta$, denoted by $\alpha \leq \beta$.
Definition 3.5 Let $M_{A}=\left(\alpha_{1}, \alpha_{2}, \cdots, \alpha_{n}\right)^{T}$ and $M_{B}=\left(\beta_{1}, \beta_{2}, \cdots, \beta_{n}\right)^{T}$, be two matrices, $\alpha_{i}$ and $\beta_{i}$ be row vectors respectively. If $\alpha_{i} \leq \beta_{i}$, we say $M_{A}$ is less than $M_{B}$, denoted by $M_{A} \leq M_{B}$.

By the definitions, dominance matrices have the following properties straightly.

Proposition 3.2 Let $\mathcal{I}^{\succeq}=(U, A \cup D, F, G)$ be an ordered information system, and $B \subseteq A$. If $M_{A}$ and $M_{B}$ are the dominance matrices, then $M_{A} \leq M_{B}$.

\subsection{Theories of Matrix Computation for Upper Approximation Reduction}

In the following, we will give the theory of matrix computation for upper approximation reduction in ordered information systems.

Theorem 3.1 Let $\mathcal{I}^{\succeq}=(U, A \cup D, F, G)$ be an information system, $B \subseteq A$, then $B$ is an upper approximation consistent set if and only if $M_{B} \leq M_{D}$.Proof “ $\Rightarrow$ ” quad we need prove that if $\overline{\eta_{B}^{\succ}}=\overline{\eta_{A}^{\succ}}$ holds for $B \subseteq A$ then $M_{B} \leq M_{D}$. So we only prove $r_{i j}=1$, when $m_{i j}=1$. In fact, we can have that

$$
m_{i j}=1 \Rightarrow x_{j} \in\left[x_{i}\right]_{B}^{\succ} \Rightarrow\left[x_{j}\right]_{B}^{\succ} \subseteq\left[x_{i}\right]_{B}^{\succ}
$$

One can obtain that if $x_{i} \notin \overline{R_{A}^{\succ}}\left(D_{k_{0}}\right)$ then $x_{j} \notin \overline{R_{A}^{\succ}}\left(D_{k_{0}}\right)$ for $k_{0} \in\{1,2, \cdots, r\}$ That is to say $x_{i} \notin \overline{R_{A}^{\succ}}\left(D_{k_{0}}\right)$ and $x_{i} \notin \overline{R_{A}^{\succ}}\left(D_{k_{0}}\right)$ don not hold at same time. Hence, we have $r_{i j}=1$.

“ $\Leftarrow$ ” Suppose $B$ be not an upper approximation consistent set, then there must exist some

$$
D_{k_{0}} \in U / R_{D}^{\succ}, \quad\left(k_{0} \in\{1,2, \cdots, r\}\right)
$$

such that

$$
\overline{R_{A}^{\succ}}\left(D_{k_{0}}\right) \neq \overline{R_{B}^{\succ}}\left(D_{k_{0}}\right) .
$$

That is to say there $x_{i} \notin \overline{R_{A}^{\succeq}}\left(D_{k_{0}}\right)$, but $x_{i} \in \overline{R_{B}^{\succeq}}\left(D_{k_{0}}\right)$. So we have

$$
\left[x_{i}\right]_{A}^{\succ} \cap D_{k_{0}}=\phi \text { and }\left[x_{i}\right]_{B}^{\succ} \cap D_{k_{0}} \neq \phi .
$$

On the other hand, we know that $\left[x_{i}\right]_{A}^{\succeq} \subseteq\left[x_{i}\right]_{B}^{\succ}$. So there exists $x_{j} \in\left(x_{i}\right)_{B}^{\geq}$and $x_{j} \in D_{k_{0}}$. Since

$$
x_{j} \in\left[x_{j}\right]_{A}^{\succ} \text {, so }\left[x_{j}\right]_{A}^{\succ} \cap D_{k_{0}} \neq \phi \text {. }
$$

Thus

$$
x_{j} \in \overline{R_{A}^{\succ}}\left(D_{k_{0}}\right) .
$$

From above, we have

$$
x_{i} \notin \overline{R_{A}^{\succ}}\left(D_{k_{0}}\right) \text { and } x_{j} \in \overline{R_{A}^{\succ}}\left(D_{k_{0}}\right) .
$$


That means $r_{i j}=0$. Since $M_{B} \leq M_{D}$, so we have $m_{i j}=0$. However, we have obtained $x_{j} \in\left[x_{i}\right]_{B}^{\succeq}$, which is a contradiction with $m_{i j}=0$.

Hence, $B$ is an upper approximation consistent set of $I^{\succeq}$ if $M_{B} \leq M_{D}$.

The theorem is proved.

Corollary 3.1 Let $\mathcal{I}^{\succeq}=(U, A \cup D, F, G)$ be an ordered information system, and $B \subseteq A . B$ is a upper approximation reduction of $I$ if and only if

$$
M_{B} \leq M_{D} \text { and } M_{B^{\prime}} \leq M_{D}
$$

does not hold for all proper subset $B^{\prime}$ of $B$.

\subsection{Algorithm of Matrix Computation for Upper Approximation Reduction}

Let $\mathcal{I}^{\succeq}=(U, A \cup D, F, G)$ be an ordered information system. We denote the dominance matrix of attributes sets $B$ by $M_{B}=\left(\beta_{1}, \beta_{2}, \cdots, \beta_{n}\right)^{T}$, and upper approximation decision matrix of $I$ by $M_{D}=\left(\gamma_{1}, \gamma_{2}, \cdots, \gamma_{n}\right)^{T}$, where $\beta_{i}, \gamma_{i}$ is the $i$ th row vectors of $M_{B}$ and $M_{D}$ respectively, and $X^{T}$ means transposed matrix of matrix $X$. So we can obtain the following algorithm by Theorem 3.1 and Corollary 3.1.

Algorithm Algorithm of matrix computation for upper approximation reduction in inconsistent ordered information systems is described as follows:

Input: An inconsistent ordered information system $\mathcal{I}^{\succeq}=(U, A \cup D, F, G)$, where $U=\left\{x_{1}, x_{2}, \cdots, x_{n}\right\}$ and $A=\left\{a_{1}, a_{2}, \cdots, a_{p}\right\}$.

Output: Upper approximation reductions of $\mathcal{I}^{\succeq}=(U, A \cup D, F, G)$.

Step 1 . Simplify the system by combining the objects with same values of every attribute.

Step 2. Calculate upper approximation decision matrix of $\mathcal{I}^{\succeq}: M_{D}=\left(\gamma_{1}, \gamma_{2}, \cdots, \gamma_{n}\right)^{T}$.

Step 3. For all $a_{l} \in A,(1 \leq l \leq p)$, calculate the first order dominance matrices,

$$
M_{\left\{a_{l}\right\}}=M_{\left\{a_{l}\right\}}^{(1)}=\left(\tau_{1}^{(1)}, \tau_{2}^{(1)}, \cdots, \tau_{n}^{(1)}\right)^{T} .
$$

For $i=1$ to $n$.

If $0 \neq \tau_{i}^{(1)} \leq \gamma_{i}$, then let $\tau_{i}^{(1)}=0$,

Denote the new matrix by $F M_{\left\{a_{l}\right\}}^{(1)}$, and turn into next step.

Step 4. Call matrix $F M_{\left\{a_{l}\right\}}^{(1)}=\left(\tau_{1}^{(1)}, \tau_{2}^{(1)}, \cdots, \tau_{n}^{(1)}\right)^{T}$,

$a_{l} \in A,(1 \leq l \leq p)$ to be the first order upper approximation matrix. If $F M_{\left\{a_{l}\right\}}^{(1)}=0$, then obtain an the first order upper approximation reduction: $\left\{a_{l}\right\}$. Otherwise, turn into next step.

Step 5. Calculate the intersection of all the first order nonzero matrix which are obtained in step 3, and call new matrices to be the second order dominance matrices, denoted by

$$
M_{\left\{a_{l} a_{s}\right\}}^{(2)}, \quad\left(M_{\left\{a_{l} a_{s}\right\}}^{(2)} \neq M_{\left\{a_{l}\right\}}^{(1)}, M_{\left\{a_{l} a_{s}\right\}}^{(2)} \neq M_{\left\{a_{s}\right\}}^{(1)}\right) .
$$

Go back to step 3 and calculate all the second order upper approximation reductions.

Step 6. Obtain the higher order upper approximation reductions by repeating step 5 . If the new matrices are zero matrices, then output all upper approximation reductions and terminate the algorithm.

From the above algorithm, we can know that the complication of times is $O\left(\left|U^{2}\right| 2^{|A|}\right)$ easily.

\section{An Example}

Example Given an ordered information system in the following Table.

From the table, we can compute the dominance matrices and upper approximation decision matrices, which are

$$
M_{\left\{a_{1}\right\}}=\left[\begin{array}{llllll}
1 & 1 & 1 & 1 & 1 & 1 \\
0 & 1 & 0 & 0 & 1 & 1 \\
1 & 1 & 1 & 1 & 1 & 1 \\
0 & 1 & 0 & 1 & 1 & 1 \\
0 & 1 & 0 & 0 & 1 & 1 \\
0 & 1 & 0 & 0 & 1 & 1
\end{array}\right]
$$$$
M_{\left\{a_{2}\right\}}=\left[\begin{array}{llllll}
1 & 1 & 0 & 0 & 1 & 1 \\
1 & 1 & 0 & 0 & 1 & 1 \\
1 & 1 & 1 & 1 & 1 & 1 \\
1 & 1 & 1 & 1 & 1 & 1 \\
0 & 0 & 0 & 0 & 1 & 0 \\
1 & 1 & 0 & 0 & 1 & 1
\end{array}\right]
$$

$$
M_{\left\{a_{3}\right\}}=\left[\begin{array}{llllll}
1 & 1 & 1 & 1 & 1 & 1 \\
0 & 1 & 1 & 1 & 1 & 1 \\
0 & 1 & 1 & 1 & 1 & 1 \\
0 & 0 & 0 & 1 & 0 & 1 \\
0 & 1 & 1 & 1 & 1 & 1 \\
0 & 0 & 0 & 1 & 0 & 1
\end{array}\right]
$$

$$
M_{D}=M_{\{d\}}=\left[\begin{array}{llllll}
1 & 1 & 1 & 1 & 1 & 1 \\
1 & 1 & 1 & 1 & 1 & 1 \\
1 & 1 & 1 & 1 & 1 & 1 \\
0 & 0 & 0 & 1 & 0 & 1 \\
1 & 1 & 1 & 1 & 1 & 1 \\
0 & 0 & 0 & 0 & 0 & 1
\end{array}\right]
$$

By comparing matrices $M_{\left\{a_{1}\right\}}, M_{\left\{a_{2}\right\}}, M_{\left\{a_{3}\right\}}$ and $M_{\{d\}}$, we can find that vectors of the first, second, third, and 5th row in matrix $M_{\left\{a_{1}\right\}}$ and $M_{\left\{a_{2}\right\}}$ are less then those in matrix $M_{\{d\}}$ respectively. So the system has not the first order upper approximation reduction. Thus the first order upper approximation matrices are as follows: 
Table 1. An ordered information system.

\begin{tabular}{lllll}
\hline$U$ & $a_{1}$ & $a_{2}$ & $a_{3}$ & $d$ \\
\hline$x_{1}$ & 1 & 2 & 1 & 3 \\
$X_{2}$ & 3 & 2 & 2 & 2 \\
$x_{3}$ & 1 & 1 & 2 & 1 \\
$x_{4}$ & 2 & 1 & 3 & 2 \\
$x_{5}$ & 3 & 3 & 2 & 3 \\
$x_{6}$ & 3 & 2 & 3 & 1 \\
\hline
\end{tabular}

$$
\begin{aligned}
F M_{\left\{a_{1}\right\}}^{(1)}= & {\left[\begin{array}{llllll}
0 & 0 & 0 & 0 & 0 & 0 \\
0 & 0 & 0 & 0 & 0 & 0 \\
0 & 0 & 0 & 0 & 0 & 0 \\
0 & 1 & 0 & 1 & 1 & 1 \\
0 & 0 & 0 & 0 & 0 & 0 \\
0 & 1 & 0 & 0 & 1 & 1
\end{array}\right] ; } \\
F M_{\left\{a_{2}\right\}}^{(1)} & =\left[\begin{array}{llllll}
0 & 0 & 0 & 0 & 0 & 0 \\
0 & 0 & 0 & 0 & 0 & 0 \\
0 & 0 & 0 & 0 & 0 & 0 \\
1 & 1 & 1 & 1 & 1 & 1 \\
0 & 0 & 0 & 0 & 0 & 0 \\
1 & 1 & 0 & 0 & 1 & 1
\end{array}\right] ; \\
F M_{\left\{a_{3}\right\}}^{(1)} & =\left[\begin{array}{llllll}
0 & 0 & 0 & 0 & 0 & 0 \\
0 & 0 & 0 & 0 & 0 & 0 \\
0 & 0 & 0 & 0 & 0 & 0 \\
0 & 0 & 0 & 0 & 0 & 0 \\
0 & 0 & 0 & 0 & 0 & 0 \\
0 & 0 & 0 & 1 & 0 & 1
\end{array}\right] ;
\end{aligned}
$$

Furthermore, the second order upper approximation matrices are

$$
\begin{aligned}
M_{\left\{a_{1}, a_{2}\right\}}^{(2)}=F M_{\left\{a_{1}\right\}}^{(1)} \cap F M_{\left\{a_{2}\right\}}^{(1)}= & =\left[\begin{array}{llllll}
0 & 0 & 0 & 0 & 0 & 0 \\
0 & 0 & 0 & 0 & 0 & 0 \\
0 & 0 & 0 & 0 & 0 & 0 \\
0 & 1 & 0 & 1 & 1 & 1 \\
0 & 0 & 0 & 0 & 0 & 0 \\
0 & 1 & 0 & 0 & 1 & 1
\end{array}\right] \\
M_{\left\{a_{1}, a_{3}\right\}}^{(2)}=F M_{\left\{a_{1}\right\}}^{(1)} \cap F M_{\left\{a_{3}\right\}}^{(1)}= & =\left[\begin{array}{llllll}
0 & 0 & 0 & 0 & 0 & 0 \\
0 & 0 & 0 & 0 & 0 & 0 \\
0 & 0 & 0 & 0 & 0 & 0 \\
0 & 0 & 0 & 0 & 0 & 0 \\
0 & 0 & 0 & 0 & 0 & 0 \\
0 & 0 & 0 & 0 & 0 & 1
\end{array}\right]
\end{aligned}
$$

$$
M_{\left\{a_{2}, a_{3}\right\}}^{(2)}=F M_{\left\{a_{2}\right\}}^{(1)} \cap F M_{\left\{a_{3}\right\}}^{(1)}=\left[\begin{array}{cccccc}
0 & 0 & 0 & 0 & 0 & 0 \\
0 & 0 & 0 & 0 & 0 & 0 \\
0 & 0 & 0 & 0 & 0 & 0 \\
0 & 0 & 0 & 0 & 0 & 0 \\
0 & 0 & 0 & 0 & 0 & 0 \\
0 & 0 & 0 & 0 & 0 & 1
\end{array}\right]
$$

So, we can see that $M_{\left\{a_{1} a_{3}\right\}}^{(2)}=M_{\left\{a_{2} a_{3}\right\}}^{(2)}$, and the 6th row vectors of them are less then those of $M_{\{d\}}$ respectively, by comparing $M_{\left\{a_{1} a_{2}\right\}}^{(2)}, M_{\left\{a_{1} a_{3}\right\}}^{(2)}, M_{\left\{a_{2} a_{3}\right\}}^{(2)}$ and $M_{\{d\}}$. Hence, we can obtain all the second order upper approximation reductions, which are $\left\{a_{1}, a_{3}\right\},\left\{a_{2}, a_{3}\right\}$.

In the next, we have

$$
F M_{\left\{a_{1}, a_{3}\right\}}^{(2)}=F M_{\left\{a_{2}, a_{3}\right\}}^{(2)}=\left[\begin{array}{cccccc}
0 & 0 & 0 & 0 & 0 & 0 \\
0 & 0 & 0 & 0 & 0 & 0 \\
0 & 0 & 0 & 0 & 0 & 0 \\
0 & 0 & 0 & 0 & 0 & 0 \\
0 & 0 & 0 & 0 & 0 & 0 \\
0 & 0 & 0 & 0 & 0 & 0
\end{array}\right]
$$

So, the algorithm is terminated.

Thus, all upper approximation reductions are $\left\{a_{1}, a_{3}\right\},\left\{a_{2}, a_{3}\right\}$ in the system of above example, which are consistent with ref.[5].

From the example, we can find that the algorithm is valid, and operated simply, for systems with a great deal of objects and attributes

\section{Conclusions}

It is well known that most of information systems are based on dominance relations because of various factors in practice. Therefore, it is meaningful to study the knowledge reductions in inconsistent ordered information system. In this article, the dominance matrix and upper approximation decision matrix are introduced in information systems based on dominance relations. Furthermore, the algorithm of upper approximation reduction is obtained, from which we can provide new approach to knowledge reductions in inconsistent systems based on dominance relations. Finally, an example illustrates the validity of this method, and shows the method is applicable to a complicated information system.

\section{References}

[1] Z. Pawlak, "Rough Sets," International Journal of Computer and Information Science, Vol. 11, No. 5, 1982, pp. 341-356. doi:10.1007/BF01001956

[2] Y. Leuang, W. Z. Wu and W. X. Zhang, "Knowledge Acquisition in Incomplete Information Systems: A Rough Set Approach,” European Journal of Operational Re- 
search, Vol. 168, No. 1, 2006, pp. 164-180. doi:10.1016/j.ejor.2004.03.032

[3] W. H. Xu and W. X. Zhang, "Measuring Roughness of Generalized Rough Sets Induced by a Covering," Fuzzy Sets and Systems, Vol. 158, No. 22, 2007, pp. 2443-2455. doi:10.1016/j.fss.2007.03.018

[4] W. X. Zhang, W. Z. Wu, J. Y. Liang and D. Y. Li, "Theory and Method of Rough Sets,” Science Press, Beijing, 2001.

[5] W. H. Xu, X. Y. Zhang and W. X. Zhang, "Upper Approximation Reduction in Inconsistent Information Systems Based on Dominance Relations," Computer Engineering, Vol. 35, No. 18, 2009, pp. 191-193.

[6] W. H. Xu, X. Y. Zhang and W. X. Zhang, "Knowledge Granulation, Knowledge Entropy and Knowledge Uncertainty Measure in Information Systems," Applied Soft Computing, Vol. 9, No. 4, 2009, pp. 1244-1251. doi:10.1016/j.asoc.2009.03.007

[7] W. H. Xu, X. Y. Zhang, J. M. Zhong and W. X. Zhang, "Attribute Reduction in Ordered Information Systems Based on Evidence Theory," Knowledge and Information Systems, Vol. 25, No. 1, 2010, pp. 169-184. doi:10.1007/s10115-009-0248-5

[8] W. H. Xu and W. X. Zhang, "Knowledge Reduction and Matrix Computation in Inconsistent Ordered Information Systems," International Journal Business Intelligence and Data Mining, Vol. 3, No. 4, 2008, pp. 409-425. doi:10.1504/IJBIDM.2008.022737

[9] W. H. Xu, M. W. Shao and W. X. Zhang, "Knowledge Reduction Based on Evidence Reasoning Theory in Ordered Information Systems," Lecture Notes in Artificial Intelligence, Vol. 4092, 2006, pp. 535-547.

[10] W. H. Xu and W. X. Zhang, "Methods for Knowledge Reduction in Inconsistent Ordered Information Systems," Journal of Applied Mathematics \& Computing, Vol. 26, No. 1-2, 2008, pp. 313-323.

[11] W. Z. Wu, M. Zhang, H. Z. Li and J. S. Mi, “Attribute Reduction in Random Information Systems Via Dempster-Shafer Theory of Evidence,” Information Sciences,
Vol. 174, No. 3-4, 2005, pp. 143-164. doi:10.1016/j.ins.2004.09.002

[12] M. Zhang, L. D. Xu, W. X Zhang and H. Z. Li, “A Rough Set Approach to Knowledge Reduction Based on Inclusion Degree and Evidence Reasoning Theory," Expert Systems, Vol. 20, No. 5, 2003, pp. 298-304. doi:10.1111/1468-0394.00254

[13] S. Greco, B. Matarazzo and R. Slowingski, "Rough Approximation of a Preference Relatioin by Bominance Relatioin,” European Journal of Operational Research, Vol. 117, No. 1, 1999, pp. 63-83. doi:10.1016/S0377-2217(98)00127-1

[14] S. Greco, B. Matarazzo and R. Slowingski, "A New Rough Set Approach to Multicriteria and Multiattribute Classificatioin. In: Rough Sets and Current Trends in Computing (RSCTC'98),” Springer-Verlag, Berlin, 1998. pp. 60-67.

[15] S. Greco, B. Matarazzo and R. Slowingski, "Rough Sets Theory for Multicriteria Decision Analysis,” European Journal of Operational Research, Vol. 129, No. 1, 2001, pp. 11-47. doi:10.1016/S0377-2217(00)00167-3

[16] S. Greco, B. Matarazzo, R. Slowingski, "Rough Sets Methodology for Sorting Problems in Presence of Multiple Attributes and Criteria,” European Journal of Operational Research, Vol. 138, No. 2, 2002, pp. 247-259. doi:10.1016/S0377-2217(01)00244-2

[17] K. Dembczynski, R. Pindur and R. Susmaga, "Generation of Exhaustive Set of Rules within DominanceBased Rough Set Approach," Electronic Notes Theory Compute Sciences, Vol. 82, No. 4, 2003.

[18] Y. Sai, Y. Y. Yao and N. Zhong, "Data Analysis and Mining in Ordered Information Tables," IEEE Computer Society Press, San Jose, 2001, pp. 497-504.

[19] M. W. Shao and W. X. Zhang, "Dominance Relation and Rules in an Incomplete Ordered Information System," International Journal of Intelligent Systems, Vol. 20, No. 2005, pp. 13-27. doi:10.1016/S0377-2217(01)00244-2 